\title{
Disturbances in intraventricular conduction in children with end-stage renal disease on peritoneal dialysis: A pilot study
}

\author{
Krystyna Laszki-Szcząchor ${ }^{1, B-E}$, Danuta Zwolińska ${ }^{2, E, F}$, Małgorzata Sobieszczańska ${ }^{1, A, D-F}$, \\ Michał Tabin ${ }^{1, B}$, Dorota Polak-Jonkisz ${ }^{2, A, B, D, F}$ \\ ${ }^{1}$ Department of Pathophysiology, Wroclaw Medical University, Poland \\ 2 Department of Pediatric Nephrology, Wroclaw Medical University, Poland \\ A - research concept and design; $\mathrm{B}$ - collection and/or assembly of data; $\mathrm{C}$ - data analysis and interpretation; \\ $D$ - writing the article; $E$ - critical revision of the article; $F$ - final approval of the article
}

Address for correspondence

Małgorzata Sobieszczańska

E-mail:malgorzata.sobieszzzanska@umed.wroc.pl

Funding sources

None declared

Conflict of interest

None declared

Received on August 20, 2016

Reviewed on November 7, 2016

Accepted on March 2, 2017

\begin{abstract}
Background. The progression of chronic kidney disease is accompanied by multi-organ disorders, among which cardiovascular diseases have the status of a serious clinical problem. The body surface potential mapping (BSPM) technique is a non-invasive method which enables the detection of pathological changes in the bioelectrical activity of the heart.
\end{abstract}

Objectives. The aim of this study was to identify possible disturbances in the intraventricular conduction system in peritoneally dialyzed children.

Material and methods. Cardiac examination consisted of 12-lead electrocardiography, echocardiography and BSPM. The evaluation of disturbances in the cardio-electrical field was performed by comparing the qualitative and quantitative features of the heart potentials on the isopotential map.

Results. Data was collected from 10 children treated with automatic peritoneal dialysis (APD) (mean age: $13.6 \pm 2.3$ years) and 26 healthy children. The maps of dialyzed children showed a shift in positive isopotentials toward the left lower part of the thorax, while negative values were observed in its left upper part. A distribution of lines on the isopotential maps revealed disturbances in the stimulation spread within the heart ventricles, especially within the anterior fascicle of the left bundle branch of His.

Conclusions. Intraventricular conduction disturbances were observed in the left bundle branch of His in the peritoneally dialyzed children. The body surface potential mapping was a more sensitive method in identifying the early stage of conduction disturbances within the heart ventricles than 12-lead electrocardiography. Further research involving a larger population of dialyzed children is planned.

Key words: children, peritoneal dialysis, isopotential maps, body surface potential mapping

DOI

10.17219/acem/69255

Copyright

Copyright by Author(s)

This is an article distributed under the terms of the

Creative Commons Attribution Non-Commercial License

(http://creativecommons.org/licenses/by-nc-nd/4.0/) 


\section{Introduction}

Patients with chronic kidney diseases (CKD) are at a high risk of cardiovascular diseases, including arrhythmia, cardiomyopathy, valve disorders, and sudden cardiac death. Cardiovascular complications become more probable with CKD progression, and are identified in $1 / 3$ of the examined dialyzed patients. ${ }^{1,2}$

Parekh et al. reported that mortality risk in children with CKD is 10 -fold higher than in the general child population, and Foley et al. stated that the mortality rate increased by even 500-1000 times. ${ }^{3-5}$ Therefore, the American Heart Association classified CKD children as the "highest risk" group. ${ }^{6}$

Numerous uremia complications in CKD children are responsible for cardiac problems, including structural changes in the myocardium and restructuring of the coronary vessels. Alterations within the intraventricular conduction system cause delayed ventricular depolarization with subsequent hemodynamic dyssynchrony and left ventricle systolic-diastolic dysfunction.

Early diagnosis of cardiovascular impairments in CKD children seems extremely beneficial, as it may offer a preventive management program which reduces the risk of morbidity and mortality from cardiovascular disorders. ${ }^{7}$ Non-invasive cardiac diagnostic techniques include body surface potential mapping (BSPM), a computerized method which enables an analysis of the cardio-electrical field activity using 3 types of maps: isopotential, isointegral and isochrones. ${ }^{8}$

Isopotential maps were used in this study, allowing the identification of initial disorders in electrical stimulation spreading through the intraventricular conduction system. Isopotential maps display the points on the thorax of equal value of heart electrical potentials connected by isolines. The identification of possible abnormalities in the cardio-electrical field of an examined patient is done by comparing the values of heart potentials at corresponding points on the reference map, enhanced by the visual evaluation of the distribution of isopotential lines.

The aim of the study was to determine the possible changes in the heart intraventricular system in children with CKD at stage 5 treated with peritoneal dialysis (PD).

To the best of our knowledge, there have been no reports so far on the identification of intraventricular heart conduction system disturbances with isopotential maps in PD children.

The present study was approved by the Research Ethics Committee of Wroclaw Medical University, Poland (No. KB - 229/2007).

\section{Material and methods}

\section{Biochemical tests}

In all patients, serum concentrations of the following substances were assayed: inorganic phosphate $\left(\mathrm{P}_{\mathrm{i}}\right)$, total calcium (t-Ca), sodium (Na), urea, creatinine (cr), intact parathormone $(\mathrm{iPTH}), \mathrm{C}$-reactive protein $(\mathrm{CRP})$, and albumin.

Serum $\mathrm{P}_{\mathrm{i}}, \mathrm{Na}$ and $\mathrm{t}-\mathrm{Ca}$ levels were tested by the direct potentiometry method, using ionoselective ISE electrodes, by a Konelab automatic analyzer (bioMérieux, Marcy l'Etoile, France), with the following normal ranges: $\mathrm{t}$-Ca: $8.80-10.80 \mathrm{mg} / \mathrm{dL} ; \mathrm{P}_{\mathrm{i}}$ : 4.5-5.52 $\mathrm{mg} / \mathrm{dL}$; and $\mathrm{Na}$ : 135-146 mmol/L. Serum creatinine was measured by the Jaffe method (normal range: $0.3-1.0 \mathrm{mg} / \mathrm{dL}$ ) and urea was determined by the enzymatic-colorimetric method (normal range: $10.00-45.00 \mathrm{mg} / \mathrm{dL}$ ). Intact parathormone was assayed by the immunoenzymatic method (enzymelinked immunosorbent assay - ELISA; Roche Diagnostics, Warszawa, Polska) (normal value: $11.00-67.00$ pg/mL). We used method for the serum albumin determination with bromocresol green solution (normal range: 3.4-4.2 g/dL).

End-stage renal disease (ESRD) children were kept on a phosphate-poor diet (phosphate intake $<500-800 \mathrm{mg}$ / /day) and were treated with compounds which bind phosphate in the intestine (calcium carbonate $<35-200 \mathrm{mg} / \mathrm{kg}$ / /day) in order to maintain a serum phosphate concentration of 4.5-5.52 $\mathrm{mg} / \mathrm{dL}$. Dietary calcium intake was defined as 500-600 mg of elemental calcium per day to keep a serum calcium concentration of $8.8-10.8 \mathrm{mg} / \mathrm{dL}$.

Measurements of blood pressure were taken after the completion of nocturnal dialysis, according to the obligatory recommendations (the Fourth Report on the diagnosis, evaluation and treatment of high blood pressure in children and adolescents from the National High Blood Pressure Education Program Working Group on High Blood Pressure in Children and Adolescents). ${ }^{9}$ The obtained RR results were assessed basing on percentile charts in relation to gender, age and body height.

Blood samples were drawn from peripheral veins of the patients after an overnight fast. The collected samples were clotted for $30 \mathrm{~min}$ and centrifuged at $4^{\circ} \mathrm{C}$ for $10 \mathrm{~min}$. The serum was stored at $-20^{\circ} \mathrm{C}$ until assayed.

The results of the biochemical analysis are presented in Table 1.

\section{ECG and ECHO}

Standard 12-lead electrocardiography (ECG) examination was performed twice in each examined child and the results were interpreted according to the commonly accepted criteria. ${ }^{10}$

In all of the patients on automatic peritoneal dialysis (APD), a transthoracic echocardiographic examination (ECHO) was carried out on the same day as BSPM using 
Table 1. The results of the biochemical analysis in the study groups

\begin{tabular}{|c|c|c|c|c|c|c|c|c|}
\hline \multicolumn{2}{|c|}{ Index group } & $\begin{array}{c}\text { Urea } \\
{[\mathrm{mg} / \mathrm{dL}]}\end{array}$ & $\begin{array}{c}\mathrm{cr} \\
{[\mathrm{mg} / \mathrm{dL}]}\end{array}$ & $\begin{array}{c}\mathrm{t}-\mathrm{Ca} \\
{[\mathrm{mg} / \mathrm{dL}]}\end{array}$ & $\begin{array}{c}\mathrm{P}_{\mathrm{i}} \\
{[\mathrm{mg} / \mathrm{dL}]}\end{array}$ & $\begin{array}{c}\mathrm{Na} \\
{[\mathrm{mmol} / \mathrm{L}]}\end{array}$ & $\begin{array}{c}\text { iPTH } \\
{[\mathrm{pg} / \mathrm{mL}]}\end{array}$ & $\begin{array}{l}\text { Albumins } \\
\text { [g/dL] }\end{array}$ \\
\hline \multicolumn{2}{|c|}{ (PD patients) $n=10$} & $95.32 \pm 7.12$ & $6.71 \pm 1.00$ & $9.35 \pm 1.35$ & $5.89 \pm 0.33$ & $138.77 \pm 3.19$ & $556.44 \pm 399.14$ & $4.02 \pm 0.28$ \\
\hline \multicolumn{2}{|c|}{$\begin{array}{l}\| \\
(\text { controls) } n=26\end{array}$} & $29.11 \pm 9.45$ & $0.73 \pm 0.08$ & $10.03 \pm 1.11$ & $4.78 \pm 0.9$ & $140.44 \pm 2.92$ & $26.3 \pm 8.98$ & $4.1 \pm 0.11$ \\
\hline \multirow{2}{*}{ p-value } & $M-W$ & $p<0.001$ & $p<0.001$ & NS & $p<0.001$ & NS & $p<0.001$ & NS \\
\hline & Student's t test & $p<0.001$ & $p<0.001$ & NS & $p<0.001$ & NS & $p<0.001$ & NS \\
\hline
\end{tabular}

PD - peritoneal dialysis; cr - creatinine; t-Ca - total calcium; $\mathrm{P}_{\mathrm{i}}$ - inorganic phosphate; Na - sodium; iPTH - intact parathormone; M-W - Mann-Whitney test; NS - nonsignificant.

a Hewlett Packard Sonos 1000 device (Hewlett Packard, Palo Alto, USA), and according to the guidelines of the American Society of Echocardiography. ${ }^{11}$

\section{Body surface potential mapping}

Changes in the bioelectrical field were monitored by the body surface potential mapping (BSPM) method with the use of an HPM 7100 system (Fukuda-Denshi Co., Tokyo, Japan), recording ECG signals simultaneously from 87 leads. Standardized, disposable electrodes were placed on the patient's body, distributed across 13 self-adhesive strips, with 7 electrodes on each strip placed along the axillary lines (Fig. 1). Such a cylindrical configuration of the electrodes enables the recording of electrocardiographic signals from the entire thoracic surface. ${ }^{12}$

Isopotential maps were plotted for the QRS ventricular complex by linking the points which show the potentials of the same value with a solid line (i.e., isolines). The identification of disorders in the stimulation spreading across

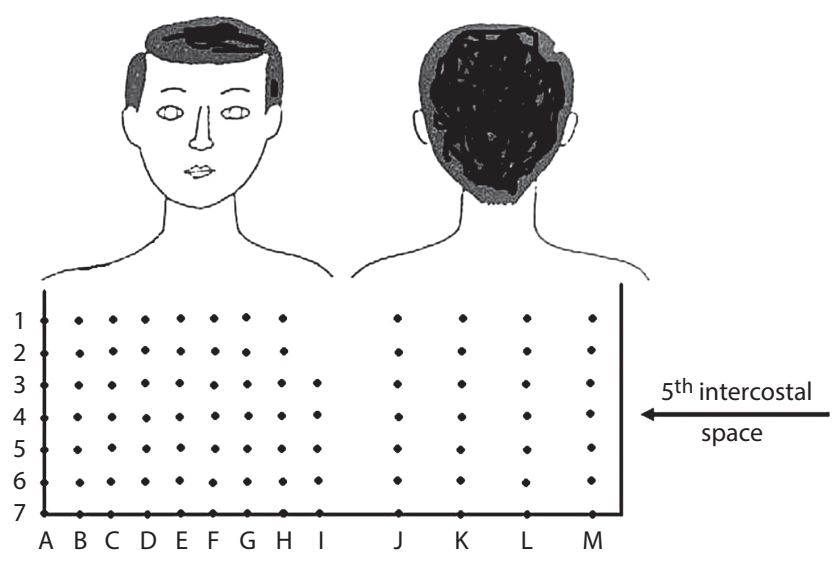

Fig. 1. The layout of electrodes on a patient's body

the intraventricular conduction system was carried out by comparing the potential values at certain points of the patient's map with the potential values at corresponding points on the reference map (averaged for healthy persons from the control group).

All of the parents of the examined children and adolescent minors enrolled in the study gave their informed consent to their child's participation in the procedures. The investigation protocol was approved by the local ethics committee and was compliant with the Declaration of Helsinki.

\section{Statistical analysis}

The data was presented as arithmetic means with standard deviation $(x \pm S D)$. The statistical significance of the null hypothesis for the equality of means expected for particular characteristics in groups I and II was verified by the Mann-Whitney nonparametric test. A p-value of $<0.05$ was considered statistically significant.

\section{Results}

The study group (group I) was comprised of 10 children with end-stage renal disease (ESRD) (4 boys and 6 girls; mean age: $13.6 \pm 2.31$ years) treated with APD. The control group (group II) consisted of 26 age-matched healthy children (12 boys and 14 girls) with no clinical renal or cardiac symptoms, and arterial blood pressure, 12-lead ECG, ECHO, and BSPM examination results within norms.

The causes of CKD in the studied group included chronic glomerulonephritis (6 patients), chronic pyelonephritis (3 patients) and congenital malformations of the urinary system (1 patient).

The APD duration varied in the studied patients from 6 months to 1.7 years (mean duration: $1.17 \pm 0.33$ years). The Kt/V index was $2.11 \pm 0.05$. The standard prescription of APD (HomeChoice Pro; Baxter Healthcare, Warszawa, Poland) included dwell changes of 9-12h, with $800-1000 \mathrm{~mL} / \mathrm{m}^{2}$ body surface area (BSA) of $1.36 \%$ glucose PD4 (Baxter Healthcare) per dwell per fill volume. Two patients had a routine APD prescription. Three patients had PD with Physioneal on alternate days; in those patients, ventricular activation time (VAT) maps were taken on the days without Physioneal. Five patients had APD cycles with $1.36 \% \mathrm{PD}$ at night and, additionally, up to 2 continuous ambulatory peritoneal dialysis (CAPD) changes during the daytime with $2.27 \%$ glucose. Glucose concentrations were dependent on the fluid amounts to be removed in order to maintain a clinically euvolemic state. Three children 
with APD were anuric. Seven other patients with residual renal function had a urine output of $721 \pm 133 \mathrm{~mL} /$ day.

The pharmacotherapy which was administered included hypotensive agents, erythropoietin, analogs of vitamin D, vitamin $\mathrm{C}$, folic acid, and iron supplements.

Six of the ESRD patients were treated with antihypertensive drugs (calcium channel blockers, angiotensinconverting enzyme inhibitors [ACEI], and beta-blocker agents). Three patients were treated with monotherapy (calcium channel blockers or ACEI) and the remaining 3 with combined therapy (calcium channel blockers with beta blockers or calcium channel blockers with ACEI).

The analysis of the distribution of isopotential lines was first performed in the control group of healthy subjects using the mean map plotted for that group (Fig. 2). In healthy subjects, isolines build up a hat-like shape on the mean map, spreading out fairly uniformly to the left and right side of the thorax. It is clearly evident that the subendocardial layer at the lower left surface of the interventricular septum and a part of the free wall of the left ventricle are the earliest stimulated parts of the heart.

In the subsequent phase of depolarization, the front of the stimulation wave moves from the left side toward the right side of the septum surface. Further on, synchronic

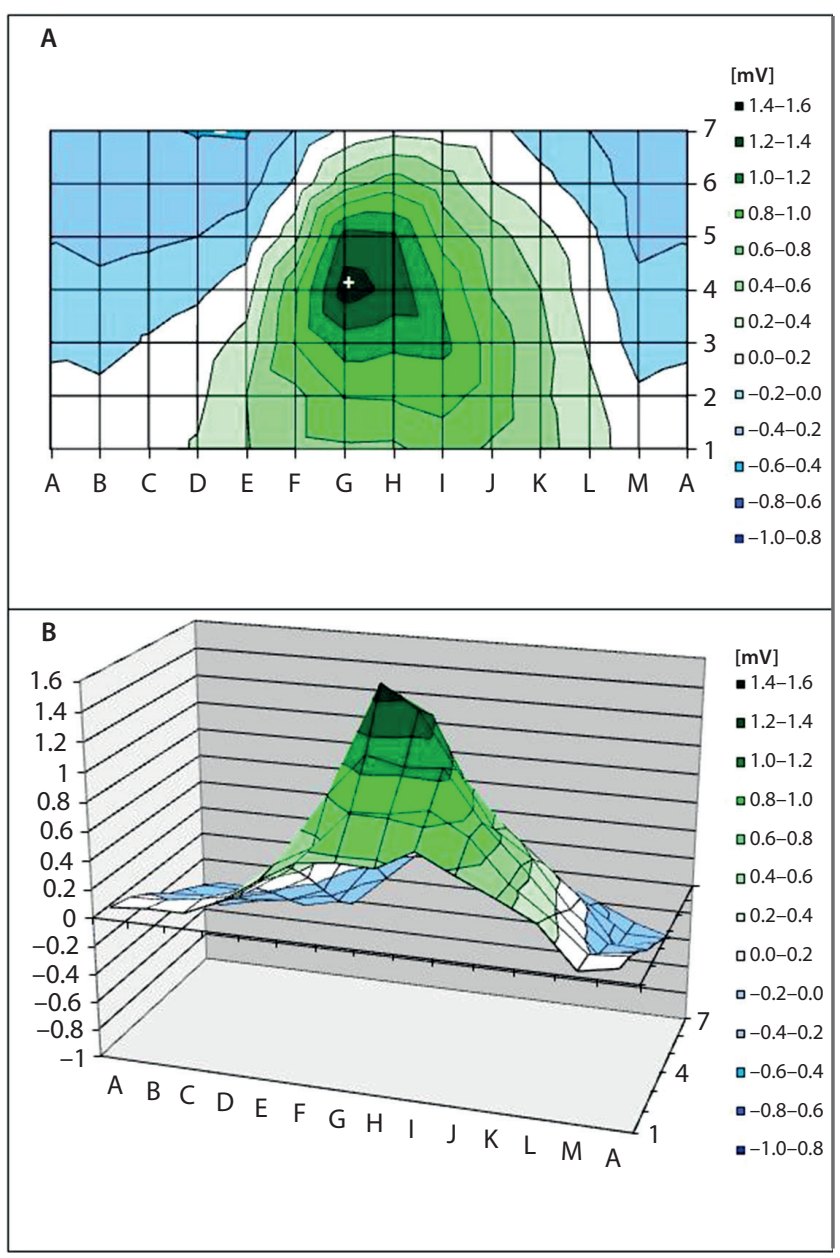

Fig. 2. Averaged isopotential map of healthy children

A - 2-dimensional; B - 3-dimensional. stimulation of the myocardium occurs, spreading out through both branches of the bundle of His.

The maximum positive potential accumulates at the G4 electrode $(1.5 \mathrm{mV})$, located in the middle of the midclavicular line in the left anterior part of the thorax. In electrocardiographic records, this area is characterized by the largest $\mathrm{R}$ waves in the QRS complex.

The stimulation wave spreads uniformly to the right and to the left side, which is depicted by the isolines that symmetrically fall on either side, i.e., to the right and to the left with decreasing values of the positive potentials.

Another phase of depolarization is identified on the epicardial surface of the right ventricle, including its para-apical and medial parts. Because of the larger thickness of the left ventricle, it arrives at the epicardial part a bit later.

The picture of the final phase of the spreading stimulation wave presents an upward direction of the isolines (negative potential values), visible on the mean map both on the right side of the thorax and on the shoulders.

The smallest registered potentials are seen mainly in the upper right part of the thorax, including the E7 and D7 electrodes $(-0.42 \mathrm{mV}$ and $-0.41 \mathrm{mV}$, respectively) at the sternal line and the right parasternal line, and in the upper part of the shoulders in the right posterior axillary line (from $-0.4 \mathrm{mV}$ to $-0.32 \mathrm{mV}$ ). Electrocardiographic records in this area present mainly QS complexes (without the $\mathrm{R}$ wave).

The distribution of isopotential lines described on the mean map for young healthy subjects is a mapped representation of the normal stimulation spread through the heart.

In turn, the distribution of isolines on the mean map plotted for the ESRD patients treated with PD (Fig. 3) only resembles the course of isolines on the map of healthy subjects in certain regions. In the PD patients, the isolines for positive potential values are initially distributed within an area similar to the one for normal subjects. However, by the left upper part of the thorax, the isolines already begin a shift toward the left lower part the thorax. The middle of the bundles, made up of positive and negative points linking the lines, is visible at the F4 electrode (the range of potential values is from $-0.04 \mathrm{mV}$ to $0.02 \mathrm{mV}$ ).

In the subsequent time interval, the patients' mean map reveals an accumulation of lines at the region between the E4, F4 electrodes along the sternal and the parasternal lines up to the G5, H5 electrodes along the midclavicular line and the left anterior axillary line.

The direction of movement of positive potential isolines clearly indicates a block in the intraventricular conduction route within the anterior fascicle of the left branch of the bundle of His. Potential values are lower than those on the map for the control group. A maximum value $(+1.13 \mathrm{mV})$ stabilizes at the $\mathrm{H} 3$ electrode at the left anterior axillary line.

Weakening positive potentials indicate a problem with the stimulation wave spreading throughout a blocked area, 


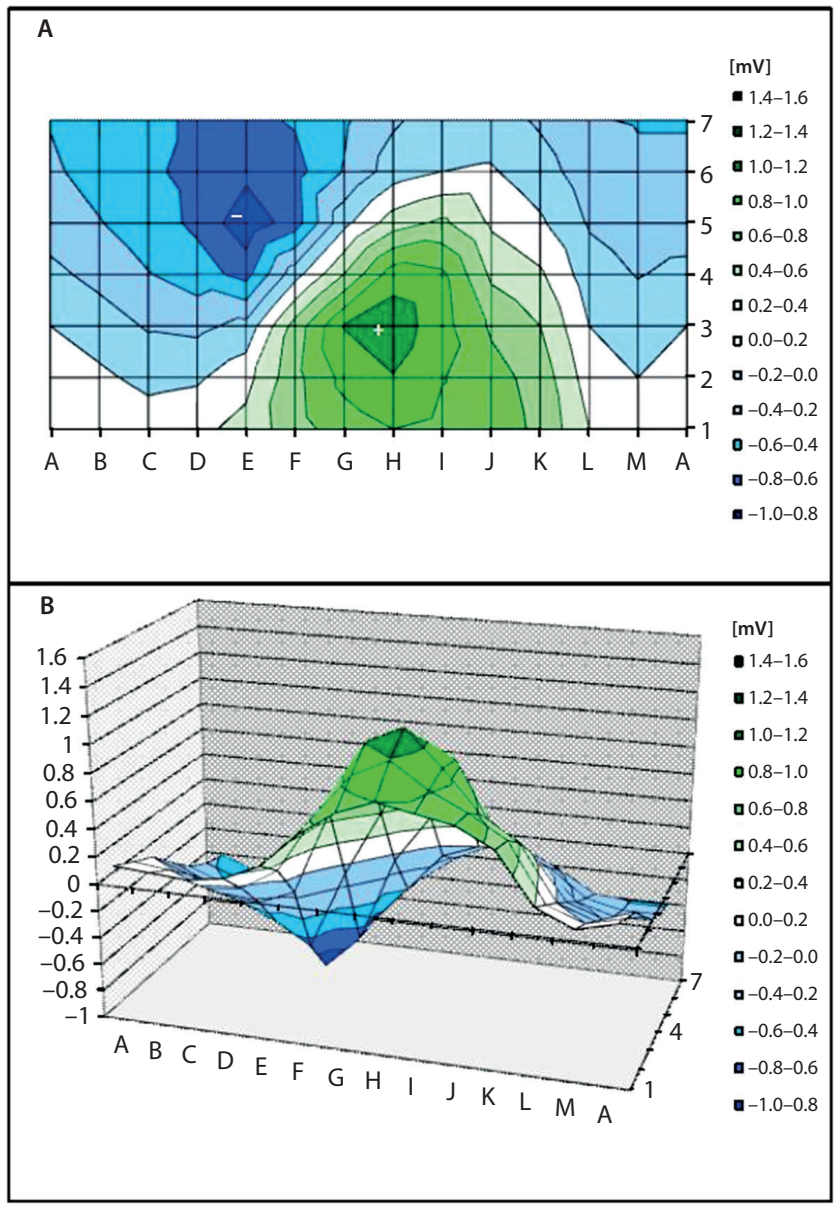

Fig. 3. Averaged isopotential map of peritoneally dialyzed children

A - 2-dimensional; B - 3-dimensional.

in which case the front of the wave tries to find any possible bypass routes. In consequence of the block at the anterior left fascicle of the bundle of His, the free wall of the posterior ventricle is stimulated with some delay via the route through the posterior bundle.

The final part of the depolarization process is observed in the right upper part of the thorax and depicted on the map with isolines of negative values. The minimum value $(-0.95 \mathrm{mV})$, also lower than in the control group, is visible at the E5 electrode in the sternal line, in the anterior part of the thorax.

The above-presented pattern of isoline distribution, which may be observed on isopotential maps of all the studied patients, is characteristic of an anterior bundle block in the left branch of the bundle of His (left anterior fascicle block [LAFB]).

No such changes were observed on standard ECG tracings, though they may simply have been beyond the 12-lead ECG capture capacity.

Regarding ECHO examination, no signs of left ventricular hypertrophy were found in the examined patients on APD, as neither left ventricular mass (LVM) nor left ventricular mass index (LVMi) exceeded the $95^{\text {th }}$ percentile, following the standards recommended by Khoury et al. ${ }^{13}$
The ECHO results also showed fairly normal left ventricle systolic function, as determined by ejection fraction (EF) and shortening fraction (FS).

\section{Discussion}

In 1998, the National Kidney Foundation Task Force on Cardiovascular Disease announced an epidemic of cardiovascular disease in patients with ESRD. ${ }^{14}$ During the subsequent years of continued observation, high mortality rates were recorded for cardiac reasons (cardiac death) not only in adult patients but also in children on renal replacement therapy. It was additionally established that the incidence of cardiac disorders did not depend on the type of dialysis therapy (hemodialysis - HD or PD), while kidney transplantation did support the normalization of cardiovascular changes, despite the administration of aggressive pharmacotherapy ${ }^{15,16}$ Following the data of the United States Renal Data System (USRDS) (2011), in patients with successful kidney transplantation, cardiac disorders are the second most common cause of death after infections. ${ }^{6}$

The application of multi-electrode ECG recording in the BSPM system has opened a new possibility for the early diagnosis of changes in the depolarization wave spreading across the heart of dialyzed patients. This process may be measured through the magnitude of potentials and through the specificity of isoline distribution on isopotential maps.

In the present study, the analysis of the isopotential maps in the examined group of peritoneally dialyzed children demonstrated a certain similarity with the control group regarding the initial phase of ventricular depolarization. This means that the stimulation process in the heart began at the same locations and time points as in healthy children. However, significant differences were observed in the further course of the stimulation process. In ESRD children on APD, a combined leftward and downward shift of the isolines began by the left upper part of the thorax, which is a clear illustration of an electrical impulse passage block in the anterior fascicle of the left branch of the bundle of His. The picture of suppressed positive potential of the electrical impulse, as observed on the isopotential maps, is direct evidence for pathological spreading of the stimulation wave within the blocked area, confirmed by the attempts of the wave front to find another, more convenient bypass.

It was clearly demonstrated in the group of peritoneally dialyzed children that the free wall of the left ventricle is stimulated with some delay through the posterior bundle of His, which results from the anterior left bundle branch block.

The pathogenesis of cardiovascular changes in children and adolescents with CKD is multifactorial, where disorders related to the primary disease and the type of therapy applied are of major significance. All these components lead to degenerative changes in cardiomyocytes, affecting 
the vascular architecture and causing strains and remodeling of the left ventricle as well as calcification in the entire myocardium.

The effects of these degenerative processes include conduction disorders in the intraventricular conduction system. ${ }^{1,5,7,15,16}$

Parathormone $(\mathrm{PTH})$ is one of the major causative factors responsible for cardiac pathologies in this population of patients. This uremic toxin is - starting in the earliest stages of CKD - significantly responsible for metabolic disorders. Following many authors, one should perceive $\mathrm{PTH}$ as a direct cause of impaired processes in the systolicdiastolic function of the heart in patients with CKD. ${ }^{17-19}$

It should be considered fairly symptomatic that high iPTH levels (also observed in our patients) support mitochondrial damage in cardiomyocytes, which in turn disturbs the energy processes in these cells. ${ }^{1,12,20}$ Moreover, PTH shares some responsibility for the excessive influx of calcium ions into the cells, leading to suppressed or even cut-off conduction of the electrical impulse among the cardiac cells. It may therefore be assumed that the decrease of the positive electrical potential observed on the isopotential maps of dialyzed children is a manifestation of disorders in the generation and spreading of the electrical stimulation signal within the cardiac ventricles, where the anterior left fascicle block causes a delay in the stimulation of the free wall of the left ventricle.

Chronic kidney disease is characterized by the retention of inorganic phosphorus, which is assumed to play a key role in the pathogenesis of disorders, such as renal osteodystrophy or circulation diseases. Unfortunately, despite the administered therapies with agents which bind phosphates in the intestines, even such agents as calcimimetics, high serum phosphate levels are observed in approx. 30\% of dialyzed patients. As is known, high P levels bind with increased serum PTH concentrations resulting from deficits of vitamin $\mathrm{D}_{3}$ and, in consequence, are associated with blood vessel calcification and elevated morbidity and mortality rates. ${ }^{21,22}$ Both inorganic phosphates and their esters are significant regulators of calcification processes in cells of the smooth muscles and cardiac valves. ${ }^{13,22,23}$ The process of cardiac calcification was identified in 2003 as a risk factor for cardiovascular disease. Cardiac calcification is thought to be strongly correlated with a predisposition to the formation of atherosclerotic coronary plaque. On the other hand, coronary artery calcification is significantly accelerated in patients with CKD, being inversely proportional to the glomerular filtration rate. ${ }^{24,25}$ Moreover, it has also been reported that the intensification of coronary calcification in CKD patients is higher than in non-CKD patients with diagnosed ischemic heart disease and, furthermore, that this factor can be regarded as an independent predictor of mortality in patients with CKD. ${ }^{26}$

It seems that in the ESRD children taking part in our study, hyperphosphatemia also contributes to the observed disorders of the spreading of electrical stimulation, compromising the conduction of the electrical impulse in a uremic heart. It should therefore be assumed that the increased concentrations of serum P observed in our patients was partly responsible for the delayed spread of the stimulation wave in the heart, as observed on the isopotential maps.

As Wang reported, there is growing evidence that the significant disorders of calcium metabolism, which support the calcification of blood vessels, may be a much stronger trigger of cardiac changes than hyperphosphatemia in patients with CKD. ${ }^{23}$ Both low and high Ca concentrations in the serum of the patients in this population may be responsible for the development of cardiovascular disorders. Moreover, $\mathrm{Ca}$, which is a signaling molecule in the process of electrical impulse transfer, may significantly contribute to disorders in spreading such an impulse across the conduction system of a uremic heart. In the studied group of children, Ca concentrations in serum did not present any statistically significant changes compared with the group of healthy children.

Following literature data from 2012, hypoalbuminemia, which is concomitant to CKD, is one of the most frequent disorders in these patients, while the mechanism is a result of both the primary disease and the applied renal displacement therapy. ${ }^{8,17,27}$ According to Mitsnefes, hypoalbuminemia contributes to the development of cardiovascular disease in half of the dialyzed patients. ${ }^{20}$

Margetts et al. stated that the occurrence of hypoalbuminemia might be explained by primary cardiac insufficiency or vascular permeability, which disturb both the extracellular fluid balance and the peritoneal membrane transport status. ${ }^{28}$ In our group of patients, we observed only statistically insignificantly decreased serum concentrations of albumins, compared with the healthy children. Thus, hypoalbuminemia could not have influenced the transmission of electrical stimulation in the intraventricular system of the heart.

It is quite likely that other uremic toxins may also play a significant role in the pathogenesis of cardiovascular changes in patients with ESRD; however, with our current knowledge we cannot identify any specific cardiotoxic substances. ${ }^{29}$

Histopathological changes in a uremic heart also seem significant for the proper functioning of the heart conduction system. Diffuse interstitial fibrosis and the loss of cardiomyocytes, including microvascular disease, lead to some instability in myocardial metabolism. In effect, functional disorders of the heart are observed, creating conditions which may support disturbances in the flow of electrical impulses.

Summing up, the method of BSPM presented in the study allows the identification of early changes in the intraventricular conduction system of the heart in children on PD. Body surface potential mapping, due to its non-invasive character, is a highly desirable technique, applied in order to evaluate changes in the functioning of the heart, especially in young populations. Cardiac monitoring of patients 
with CKD with this method is very helpful in the prognosis of cardiac episodes, while the identification of disturbed conductivity in the left branch of the bundle of His in uremic patients demands special attention of medical staff. ${ }^{30,31}$

The rather small study group was an obvious limitation of this pilot study. It may, in some way, be justified by the objective fact that the number of children with end-stage renal failure is not large, compared with adult patients. Moreover, because of legal and formal regulations, we were only able to evaluate the children hospitalized in our hospital.

\section{Conclusions}

In peritoneally dialyzed children, intraventricular conductivity disorders were observed in the left branch of the bundle of His, as confirmed by changes seen on isopotential maps.

Body surface potential mapping is a more precise diagnostic method than standard ECG, allowing for the early identification of changes not evident on ECG recordings.

Further research involving a larger population of children with end-stage renal failure and on PD is planned in order to verify and strengthen the evidence for the preliminary findings of this study.

\section{References}

1. Chavers BM, Li S, Collins AJ, Herzog CA. Cardiovascular disease in pediatric chronic dialysis patients. Kidney Int. 2002;62:648-653.

2. Parekh RS. Expect the unexpected: Sudden cardiac death in dialysis patients. Clin J Am Soc Nephro. 2012;7:8-11.

3. Parekh RS, Carroll CE, Wolfe RA, Port FK. Cardiovascular mortality in children and young adults with end-stage kidney disease. J Pediatr. 2002;141:191-197.

4. Almeida FA, Machado FC, Moura JA Jr, Guimarães AC. Global and cardiovascular mortality and risk factors in patients under hemodialysis treatment. Arq Bras Cardiol. 2010;94:187-192,201-206,190-195.

5. Foley RN, Parfrey PS, Sarnak MJ. Clinical epidemiology of cardiovascular disease in chronic renal disease. Am J Kidney Dis. 1998;32 (Suppl 3):S112-S119.

6. US Renal Data System. USRDS 2011 Annual Data Report: Atlas of Chronic Kidney Disease and End-Stage Renal Disease in the United States. Bethesda, MD: National Institutes of Health, National Institute of Diabetes and Digestive and Kidney Diseases; 2011. https://www.usrds. org/atlas11.aspx. Accessed December 15, 2015.

7. Groothoff J, Liien M, Kar N, Wolff E, Davin J. Cardiovascular disease as a late complication of end-stage renal disease in children. Pediatr Nephrol. 2005;20:374-379.

8. Laszki-Szcząchor K, Polak-Jonkisz D, Zwolińska D, Rusiecki L, Janocha A, Sobieszczańska M. Heart ventricular activation in VAT difference maps from children with chronic kidney disease. Pediatr Nephrol. 2012;27:251-259.

9. The Fourth Report on the diagnosis, evaluation and treatment of high blood pressure in children and adolescents. National High Blood Pressure Education Program Working Group on High Blood Pressure in Children and Adolescents. Pediatrics. 2004;114:555-576.
10. Wagner G. Marriott's Practical Electrocardiography. $10^{\text {th }}$ ed. Philadelphia, PA: Lippincott Williams and Wilkins; 2001.

11. Spencer KT, Kimura BJ, Korcarz CE, Pellikka PA, Rahko PS, Siegel RJ. Focused cardiac ultrasound: Recommendations from the American Society of Echocardiography. J Am Soc Echocardiogr. 2013;26:567-581.

12. Heart Potential Mapping System. Tokyo: Fukuda Denshi Co., Ltd. Bulletin; 1990.

13. Khoury PR, Mitsnefes MS, Daniels SR, Kimball TR. Age specific reference intervals for indexed left ventricular mass index in children. $J$ Am Soc Echocardiogr. 2009;22:709-714.

14. Levey AS, Beto JA, Coronado BE, National Kidney Foundation Task Force on Cardiovascular Disease. Controlling the epidemic of cardiovascular disease in chronic renal disease: What do we know? What do we need to learn? Where do we go from here? Am J Kidney Dis. 1998;32:853-906.

15. Van Biesen W, Verbeke F, Vanholder R. Cardiovascular disease in haemodialysis and peritoneal dialysis: Arguments pro peritoneal dialysis. Nephrol Dial Transplant. 2007;22:53-58.

16. Pego C, Rodrigues A, Ronco C. Role of peritoneal dialysis as a chronic renal replacement therapy in cardiorenal patients. Contrib Nephrol. 2012;178:182-188.

17. Robinson RF, Nahata MC, Sparks E, et al. Abnormal left ventricular mass and aortic distensibility in pediatric dialysis patients. Ped Nephrol. 2005;20:64-68.

18. Mitsnefes MM, Kimball TR, Kartal J, et al. Cardiac and vascular adaptation in pediatric patients with chronic kidney disease: Role of calcium-phosphorus metabolism. J Am Soc Nephrol. 2005;16:2796-2803.

19. Wanic-Kossowska M, Lehmann P, Czekalski S. Left ventricular systolic and diastolic dysfunction in patients with chronic renal failure treated with hemodialysis. Pol Arch Med Wewn. 2003;109:365-373.

20. Mitsnefes MM. Cardiovascular disease in children with chronic kidney disease. J Am Soc Nephrol. 2012;23:578-585.

21. Cozzolino M, Stucchi A, Rizzo MA, et al. Phosphate control in peritoneal dialysis. Contrib Nephrol. 2012;178:116-123.

22. Mozos I. Laboratory markers of ventricular arrhythmia risk in renal failure. Hyperphosphatemia:Treatment options. Biomed Res Int. 2014. http://dx.doi.org/10.1155/2014/509204

23. Wang AY. Calcium balance and negative impact of calcium load in peritoneal dialysis patients. Perit Dial Int. 2014;34:345-352.

24. Russo D, Palmiero G, De Blasio AP, Balletta MM, Andreucci VE. Coronary artery calcification in patients with CRF not undergoing dialysis. Am J Kidney Dis. 2004;44:1024-1030.

25. Fox CS, Larson MG, Keyes MJ, et al. Kidney function is inversely associated with coronary artery calcification in men and women free of cardiovascular disease: The framing ham heart study. Kidney Int. 2004;66:2017-2021.

26. Iyer H, Abraham G, Reddy YN, et al. Risk factors of chronic kidney disease influencing cardiac calcification. Saudi J Kidney Dis Transpl. 2013;24:1189-1194.

27. Bosch A, Ulmer HE, Keller HE, Bonzel KE, Schärer K. Electrocardiographic monitoring in children with chronic renal failure. Pediatr Nephrol. 1990;42:140-144.

28. Margetts PJ, McMullin JP, Rabbat CG, Churchill DN. Peritoneal membrane transport and hypoalbuminemia: Cause or effect? Perit Dial Int. 2000;20:14-18.

29. Segall $L$, Covic A. Cardiovascular disease in haemodialysis and peritoneal dialysis: Arguments for haemodialysis. Nephrol Dial Transplant. 2007;22:59-63.

30. Devereux RB, Alonso DR, Lutas EM, et al. Echoradiographic assessment of left ventricular hypertrophy: Comparison to necropsy findings. Am J Cardiol. 1986;57:450-458.

31. Scharer K, Schmidt KG, Soergel M. Cardiac function and structure in patients with chronic renal failure. Pediatr Nephrol. 1999;13:951-965. 